\title{
Discrete Electro-optic Beam Steering for LIDAR
}

\author{
B. Haylock*a, M.A. Baker ${ }^{\mathrm{b}}$, T. M. Stace ${ }^{\mathrm{b}}$, M. Lobino ${ }^{\mathrm{a}, \mathrm{c}}$
}

${ }^{a}$ Centre for Quantum Dynamics, Griffith University, Brisbane, Qld, 4111, Australia; ${ }^{\mathrm{b}}$ ARC Centre of Excellence for Engineered Quantum Systems(EQUS), School of Mathematics \& Physics, University of Queensland, Brisbane, 4072, Australia; ${ }^{\circ}$ Queensland Micro- and Nanotechnology Centre, Griffith

University, Brisbane, Qld, 4111, Australia

\begin{abstract}
High performance beam steering techniques are essential for $3 \mathrm{~d}$ imaging systems, and in particular automotive LIDAR. We present a high-speed, chip-scale, dynamically reconfigurable beam steering system suitable for use at telecom wavelengths. Our technique relies on sub-microsecond electro-optic switching between discrete outputs in a waveguide network. This beam steering technology, along with a coherent detection technique is used to demonstrate simultaneous ranging and Doppler velocimetry of targets with an amplitude modulated continuous wave solid state LIDAR system in a laboratory environment.
\end{abstract}

Keywords: Lidar, Beam Steering, Electro-optic Switching

\section{INTRODUCTION}

The beam steering requirements for automotive LIDAR are challenging, requiring compact, high-speed, dynamically reconfigurable steering using infrared wavelengths. Additionally compatibility with coherent measurement protocols is highly desirable, as it lowers the peak optical power requirements of the system. The first multi-pixel commercial LIDAR systems rely on physically large spinning mirrors. Recent advances have moved to smaller systems, such as MEMS mirrors ${ }^{1}$, VCSEL's ${ }^{2}$, and optical phased arrays ${ }^{3,4}$ in an attempt to shrink system size and improve reliability. These new techniques have allowed for higher resolution sensors with faster refresh rates. However the size of the point cloud generated to be used for autonomous driving applications is still limited by scanning speed.

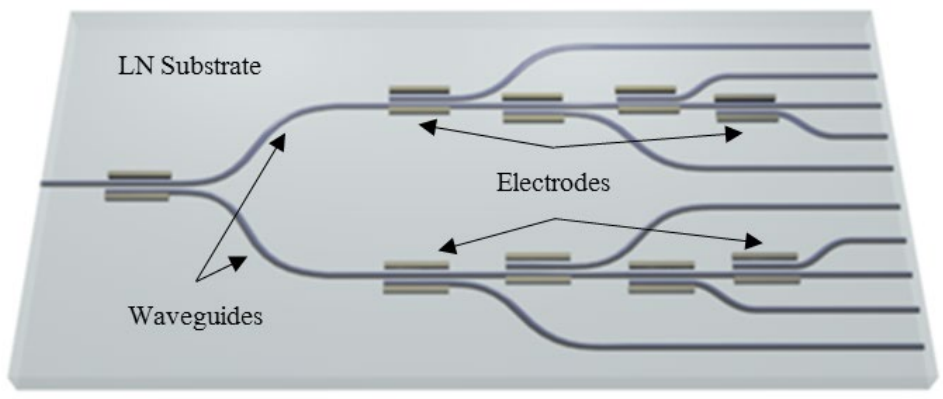

Figure 1 Schematic depiction of electro-optic switch network suitable for discrete beam steering. Details in text.

We introduce an 1:N electro-optic switch for the purposes of beam steering. This approach, rather than continuously sweeping a beam through an output angle instead, has a discrete 'point-by-point' style output, each with distinct spatial modes. A key advantage of such an approach is the ability to dynamically reconfigure the active channel of the output array, enabling software controlled beam steering for intelligent focusing on critical areas within the sensors field of view. This discrete scanning style has previously been demonstrated using the unsuitably slow thermo-optic tuning of a silicon photonic circuit ${ }^{5}$. Discrete electro-optic switching offers beam steering that is fast, solid-state, and single mode, with no side-lobes, overcoming the major technical challenges of other beam steering implementations.

As depicted in Figure 1 the electro-optic switch consists of a network of directional couplers whose splitting ratio may be tuned between 0 and $100 \%$ using electrodes patterned around the coupling region. This allows for a device which may

*b.haylock@griffith.edu.au;

AOS Australian Conference on Optical Fibre Technology (ACOFT) and Australian Conference on Optics, Lasers, and Spectroscopy (ACOLS) 2019, edited by Arnan Mitchell, Halina Rubinsztein-Dunlop, Proc. of SPIE Vol. 11200, 112001P · C 2019 SPIE · CCC code: 0277-786X/19/\$21 - doi: 10.1117/12.2539902 
deliver the light at the input to any combination of the outputs, reconfigurable in sub-microsecond timescales. The switch is fabricated in congruent lithium niobate using the reverse proton exchange process ${ }^{6,7}$, before electrodes are fabricated from sputter deposition and etching of aluminum. For this demonstration we create a switch with three output channels with a total optical loss $\sim 4 \mathrm{~dB}$. We characterise the $3 \mathrm{~dB}$ roll-off of the electrode response to be $\sim 300 \mathrm{MHz}$, improvable considerably with appropriate electrode design.

\section{LIDAR WITH DISCRETE BEAM STEERING}

We employ this discrete electro-optical beam steering technique for amplitude modulated continuous wave LIDAR. The outgoing beam is modulated with a square pulse sequence using an electro-optic modulator before passing to the beam steering chip, which is configured to steer to each output in sequence. The output is collimated by a lens onto a spinning target at a ground-truth distance of approx. $4.7 \mathrm{~m}$. The return signal is interfered with a local oscillator beam on a $3 \mathrm{~dB}$ optical coupler and then homodyne detection is performed on the two outputs.

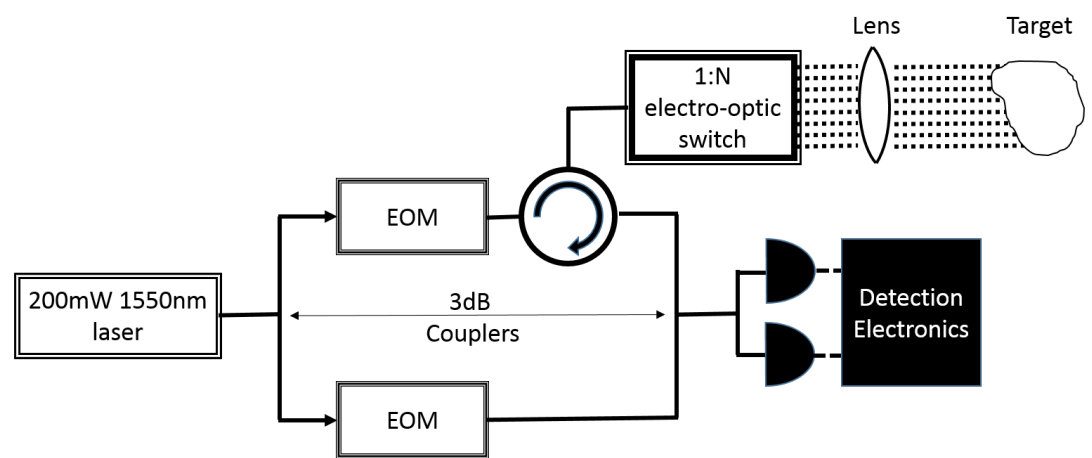

Figure 2 Schematic of amplitude modulated continuous wave LIDAR system suitable for simultaneous ranging and Doppler velocimetry. EOM - electro-optic modulator

From the output of the detection electronics, frequency domain data can be used to observe the velocity image acquired, while, with a timing reference acquired from the driving signal of the electro-optic modulator, the delay of the return signal can be used to calculate the target range from the same data simultaneously. In summary we have presented a high-speed electro-optic switch as an ideal candidate for free space beam steering applications, including automotive LIDAR. Using this beam steering technology we created a prototypical three pixel lidar system with simultaneous ranging and Doppler velocimetry. The approach we describe is scalable up to 1000 channels per chip, and with the addition of a second slower beam scanning technology across all the outputs can create a $2 \mathrm{~d}$ scanning system, one dimension discrete and the other continuous.

\section{REFERENCES}

[1] Holmström, S. T. S., Baran, U. and Urey, H., "MEMS laser scanners: A review," J. Microelectromechanical Syst. 23, 259-275 (2014).

[2] Carlson, N. W., Evans, G. A., Amantea, R., Palfrey, S. L., Hammer, J. M., Lurie, M., Carr, L. A., Hawrylo, F. Z., James, E. A., Kaiser, C. J., Kirk, J. B. and Reichert, W.F., "Electronic beam steering in monolithic gratingsurface-emitting diode laser arrays," Appl. Phys. Lett. 53, 2275-2277 (1988)

[3] Van Acoleyen, K., Rogier, H. and Baets, R. "Two-dimensional optical phased array antenna on silicon-oninsulator," Opt. Lett. 18, 265-298 (2010).

[4] Sun, J., Timurdogan, E., Yaacobi, A., Hosseini, E. S., and Watts M. R., "Large-scale nanophotonic phased array," Nature 493, 195-199 (2013).

[5] Martin, A., Bourderionnet, J., Leviander, L., Parsons, J. F., Silver, M. and Feneyrou, P., "Coherent Lidar for 3D-imaging through obscurants," in 19th Coherent Laser Radar Conference (2018), pp. 19-23.

[6] Jackel, J. L. and Johnson, J. J., "Reverse exchange method for burying proton exchanged waveguides," Electron. Lett. 27, 1360 (1991).

[7] Lenzini, F., Kasture, S., Haylock, B. and Lobino, M., "Anisotropic model for the fabrication of annealed and reverse proton exchanged waveguides in congruent lithium niobate," Opt. Express 23, 1748 (2015) 